\title{
The incidence and results of laser enhancement after cataract and refractive surgery with trifocal lens implantation
}

\author{
Drahomira Barakova ${ }^{\mathrm{a}, \mathrm{b}}$, Dana Jordanovova ${ }^{\mathrm{a}}$, Martin Sramka ${ }^{\mathrm{a}, \mathrm{c}}$, Adriana Kaluzakovaa ${ }^{\mathrm{a}}$, Martina Sajdikova ${ }^{\mathrm{a}}$
}

Purpose. To evaluate the incidence of laser enhancement following cataract surgery and refractive lens exchange (RLE) with FineVision Micro F trifocal lens implantation (PhysIOL, Liège, Belgium).

Methods. Retrospective study of patients who had undergone cataract or RLE surgery and had received a FineVision Micro F intraocular lens. Laser enhancement of residual refractive error was determined. Visual acuity (VA) assessments were performed before and after surgery: uncorrected distance VA (UCDVA), best-corrected distance VA (BCDVA), uncorrected near VA (UCNVA), plus preoperative and postoperative spherical equivalent (SE) assessments.

Results. Of the 1129 eyes from 596 patients, 61 (5.4\%) required laser enhancement to correct residual refractive error (by group: 30/679 eyes [4.4\%] cataract; 31/450 eyes [6.9\%] RLE). Eleven eyes received FemtoLASIK; 50 eyes received PRK. Mean UCDVA before laser enhancement was $0.26 \pm 0.19 \log M A R$ and $0.24 \pm 0.14$ in the FemtoLASIK and PRK groups, respectively. After laser enhancement, these were $0.04 \pm 0.05 \log M A R$ and $0.13 \pm 0.19$, respectively; BCDVA values were $0.00 \pm 0.00 \log$ MAR in the FemtoLASIK group and $0.06 \pm 0.11$ in the PRK group. Laser enhancement improved UCNVA (Jaeger) from 2-3 to 1-2 in both groups. Enhancement reduced preoperative SE of $-0.39 \pm 0.99 \mathrm{D}$ and $-0.53 \pm 0.58 \mathrm{D}$ (FemtoLASIK and PRK groups, respectively) to $0.24 \pm 0.36 \mathrm{D}$ and $0.04 \pm 0.47 \mathrm{D}$.

Conclusions. The FineVision Micro $\mathrm{F}$ trifocal lens is an effective solution for gaining increased spectacle independence. The incidence of residual refractive error requiring laser enhancement is low, and laser procedures are a safe and effective solution for improving the quality of vision and patient satisfaction.

Synopsis. Laser enhancement rates and outcomes were determined following cataract / refractive lens exchange surgery that used a trifocal IOL. Enhancement was safe and effective and rates were low (5.4\%).

Key words: trifocal IOL, laser enhancement, cataract surgery, refractive lens exchange

Received: October 28, 2020; Revised: January 15, 2021; Accepted: January 20, 2021; Available online: February 4, 2021

https://doi.org/10.5507/bp.2021.010

(c) 2022 The Authors; https://creativecommons.org/licenses/by/4.0/

${ }^{a}$ Gemini Eye Clinic, Prague, Czech Republic

${ }^{b}$ Department of Ophthalmology, Motol University Hospital, Prague, Czech Republic

'Department of Circuit theory/Faculty of Electrical engineering, Czech Technical University in Prague, Czech Republic

Corresponding author: Dana Jordanovovs, e-mail:dana.jordanovova@gemini.cz

\section{INTRODUCTION}

Cataract surgery is increasingly becoming a refractive procedure ${ }^{1}$-hence the development of "refractive lens exchange" (RLE) to correct visions in lenses that are not cataractous. This means that increasing emphasis is being placed on refractive outcomes ${ }^{2}$. Patients' demands are high; they want spectacle independence. To give patients good vision at near, intermediate and distance, a trifocal intraocular lens is a good solution for many.

Cataract surgery requires ocular biometry to be performed so the intraocular lens (IOL) selected for each patient has the correct dioptric power. Nevertheless, no system is perfect, and errors can be made (even with the most modern biometry systems) and "refractive surprises" (postoperative refractive error) can occur. Almost half of the errors in intraocular lens power calculation using ultrasound biometry are attributed to axial length measurement errors ${ }^{3}$, preoperative estimations of postoperative IOL position and determination of postoperative refraction, in a low percentage also pupil size variation and variability of IOL power".

Multiple methods can be used to correct the unexpected refractive error. IOL exchange, implantation of a piggyback lens ${ }^{5}$ or excimer laser surgery (typically laser in situ keratomileusis [LASIK] or photorefractive keratectomy [PRK]) which are a safe, effective and predictable method to correct ametropia after cataract extraction with IOL implantation ${ }^{6}$.

We aimed to evaluate the incidence of residual refractive error laser corrections, and the results of these procedures in patients who had undergone cataract surgery or RLE and who had received a trifocal IOL.

\section{METHODS}

We performed a retrospective data review of 1129 eyes of 596 patients who underwent uncomplicated cataract (679 eyes) or RLE surgery (450 eyes) and received a trifocal FineVision Micro F lens (PhysIOL, Liège, Belgium) during the period from September 2014 to June 2018.

Preoperatively, patients underwent an examination that included objective refraction and intraocular pressure measurement on an automatic refracto/keratotonometer (Tonoref II, Nidek, Aichi, Japan), optical biometry (IOLMaster 500, Carl Zeiss Meditec AG, Jena, Germany), corneal topography (Pentacam HR, 
Oculus Optikgeräte, Wetzlar, Germany), endothelial microscopy (CEM-530 specular microscope, Nidek). The uncorrected distance visual acuity (UCDVA) and the best-corrected distance visual acuity (BCDVA) were measured using Snellen optotypes, uncorrected near visual acuity (UCNVA) and best-corrected near visual acuity (BCNVA) using Jaeger optotypes. The examination also included a test of binocular function. All patients underwent slit-lamp examination, fundus examination with indirect ophthalmoscopy and optical coherence tomography (OCT, RTVue, Optovue, Inc., Fremont, CA). The choice of spherical power of the intraocular lens was based on optical biometry measurements, taking into account the SRK/T, Haigis, Holladay, and Hoffer Q patterns based on the axial length and keratometry of the patient. All eyes were targeted for emmetropia.

Exclusion criteria for implantation of the trifocal lens were the presence of ocular diseases, that are expected to affect a final VA such as retinal diseases (e.g macular degeneration), glaucoma, corneal topography irregularities, corneal diseases, pseudoexfoliation syndrome, traumatic cataract, a history of ocular inflammation, or dry eye syndrome.

\section{The primary procedure}

Cataract and RLE surgery were performed under topical anesthesia, with a $2.2 \mathrm{~mm}$ microincision. After continuous circular capsulorhexis and hydrodissection, the phacoemulsification of the nucleus, irrigation, and aspiration of the cortex was performed using the Stellaris Elite PC Vision Enhancement System (Bausch + Lomb, Rochester, NY, USA) and then the trifocal lens was implanted through the injector into the bag. Before the implantation of the lens, a $12 \mathrm{~mm}$ diameter capsular tension ring (Bio-Tech Vision Care Pvt. Ltd., Khatraj Gandhinagar, India) was implanted into the capsular bag.

The FineVision Micro F lens is a biconvex aspheric diffractive single-piece intraocular lens with a total length of $10.75 \mathrm{~mm}$, a $6.15 \mathrm{~mm}$ optical zone, $5^{\circ}$ angulation, and a $360^{\circ}$ square edge. The additional power for intermediate vision is $+1.75 \mathrm{D}$, for near vision $+3.5 \mathrm{D}$.

Postoperatively, all patients underwent an examination that included objective and subjective refraction, intraocular pressure measurement and slit-lamp examination on the first day, one week and one month after the primary procedure.

In the cases of refractive error, we repeated the examination three months after the surgery. Inclusion criteria for retreatment were patient's dissatisfaction with their visual outcomes as a result of residual refractive error that was stable for at least 3 months after the primary procedure, and/or difference of a minimum of two lines between UCDVA and BCDVA. We determined the rate of the need for laser enhancement of residual refractive error in cataract and RLE group.

The same examinations before primary procedure were done (with the exclusion of biometry). Corneal topography and the presence of dry eye (using Schirmer II and tear film break-up time tests) were also evaluated.

\section{The secondary procedure}

FemtoLASIK was performed under topical anesthesia. Corneal flaps were created by a FEMTO LDV Z6 (Ziemer Ophthalmic Systems AG, Port, Switzerland) system and laser enhancement was performed with SCHWIND AMARIS 500 excimer laser (SCHWIND eye-tech-solutions $\mathrm{GmbH}$, Kleinostheim, Germany). The treatment was based on patient's subjective refraction.

After surgery, patients applied dexamethasone-tobramycin solution (Tobradex, Alcon Laboratories Inc, Fort Worth, TX, USA) for 3-4 weeks and preservative-free artificial tears for at least three months after laser surgery.

PRK was also performed under topical anesthesia. The corneal epithelium was removed with a sponge after the instillation of alcohol for $20 \mathrm{~s}$. Laser enhancement was performed with SCHWIND AMARIS 500 laser (SCHWIND eye-tech-solutions GmbH, Kleinostheim, Germany). Again, treatment was based on patient's subjective refraction.

After surgery, a therapeutic soft contact lens was placed on the cornea, and it was removed after corneal re-epithelization was complete. Patients applied levofloxacin $5 \mathrm{mg} / \mathrm{mL}$ solution (Oftaquix, Bausch and Lomb, Milan, Italy) until the reepithelization process finished and Tobradex solution for 3 weeks. Then, they used fluorometholone (Flucon, Novartis,) for another two months. During the whole healing process, patients used preservative-free artificial tears.

After the laser surgery, UCDVA, BCDVA, UCNVA, postoperative spherical equivalent (SE) and type of laser surgery were evaluated.

\section{Study evaluation}

The data were evaluated in GraphPad Prism 8 and Microsoft Excel 2013. The Wilcoxon matched-pairs signed-rank test was used to evaluate the SE in the FemtoLASIK and PRK group and McNemar's test was used to evaluate the SE before and after laser enhancement. VA values are expressed as mean \pm standard deviation in $\log$ MAR or as a range in Jaeger notation.

\section{RESULTS}

In this study, we evaluated 1129 eyes of 596 patients, who underwent uncomplicated cataract surgery (679 eyes; $60.1 \%$ ) and an RLE surgery (450 eyes $39.9 \%$ ) with trifocal FineVision Micro F lens implantation.

Laser enhancement of the refractive error was performed in 61 eyes $(5.4 \%)$, out of which 30 eyes $(4.4 \%)$ in the cataract group and 31 eyes $(6.9 \%)$ in the RLE group. The average age of patients in the cataract group was $57 \pm 10$ years $(36 ; 75)$, in the RLE group $52 \pm 5$ years $(40$; 61 ), the male-to-female ratio in cataract group was 8:17, in the RLE group 9:11. No concomitant eye diseases were present in both groups.

Refractive errors after primary surgery were divided into three groups according to preoperative SE. There were 13 eyes in hypermetropic group and 43 eyes in the myopic group, from which all patients were in range of 
low myopia <-3.0 D SE. Five patients underwent laser enhancement because of mixed astigmatism.

The average central corneal thickness before laser surgery was $534.7( \pm 25.6)$ microns, the mean depth of laser ablation in all groups was $25.15( \pm 14.64)$ microns.

\section{Refractive outcomes}

After the laser enhancement, we reached the SE < $\pm 0.5 \mathrm{D}$ in $67 \%$ of eyes, $< \pm 1.0 \mathrm{D}$ in $92 \%$ of eyes and a SE of $< \pm 1.5 \mathrm{D}$ in $100 \%$ of eyes (Fig. 1). A scattergram shows the pre-enhancement versus post-enhancement SE (Fig. $2)$. Laser correction by FemtoLASIK was performed in $11 / 61(18.0 \%)$ eyes, the PRK method in 50/61 (82.0\%) eyes.

\section{FemtoLASIK group}

The mean UCDVA before laser enhancement was $0.26 \pm 0.19 \log$ MAR. After a laser enhancement, it reached $0.04 \pm 0.05 \log$ MAR. BCDVA achieved values of $0.00 \pm 0.00$ logMAR. UCNVA, evaluated based on Jaeger optotypes, improved from values of 2-3 before laser enhancement to 1-2 after laser enhancement. The preoperative SE of $-0.39 \pm 0.99 \mathrm{D}$ was reduced to $0.24 \pm 0.36 \mathrm{D}$ after enhancement.

\section{PRK group}

The mean UCDVA before laser enhancement was $0.24 \pm 0.14 \log$ MAR. After enhancement, it reached $0.13 \pm 0.19 \log$ MAR; BCDVA achieved values of $0.06 \pm 0.11 \log$ MAR. UCNVA, evaluated based on Jaeger optotypes, improved from values of 2-3 before laser enhancement to 1-2 after laser enhancement. The preoperative SE $-0.53 \pm 0.58 \mathrm{D}$ was reduced to $0.04 \pm 0.47 \mathrm{D}$ after enhancement (Fig.3).

\section{Retreatments}

There was a need for one additional retreatment in three out of the 61 eyes $(4.92 \%)$. One eye was in the
FemtoLASIK group, as after the first enhancement, there was a residual hypermetropia. Two eyes were in the PRK group, with the indication for retreatment being residual mixed astigmatism.

\section{DISCUSSION}

Patients who are strongly motivated to be spectacle independent are typically good candidates for receiving at trifocal intraocular lens. Multifocal IOLs do not recapitulate the accommodation of the crystalline lens; instead, they provide pseudoaccommodation where patients receive multiple images in sharp focus on their retina, and their brain "picks" the image they wish to see in a process called neuroadaptation. Recent improvements to multifocal IOL designs, such as the addition of asphericity or apodization-and good preoperative judgment in the choice of right multifocal IOL to suit the patients' needs, means that these lenses are being used successfully in clinical practice today ${ }^{7}$.

Patients' satisfaction with the outcome of surgery depends on a number of factors. Most important are their visual requirements and preferences, ocular findings, accurate measurements, that they undergo an uncomplicated surgery and receive appropriate postoperative care. Patient education and explanation of the need for neuroadaptation also play a key role.

Before any surgery occurs, the right IOL power needs to be calculated, and this power varies from patient to patient. Preoperative biometry (of corneal thickness, anterior chamber depth, lens position etc.) needs to be performed to calculate this, but there are also a number of IOL calculation formulas that exist (e.g. SRK/T, Haigis, Holladay, and Hoffer Q); some formulas can perform better than others under certain circumstances, like high myopia ${ }^{2}$.

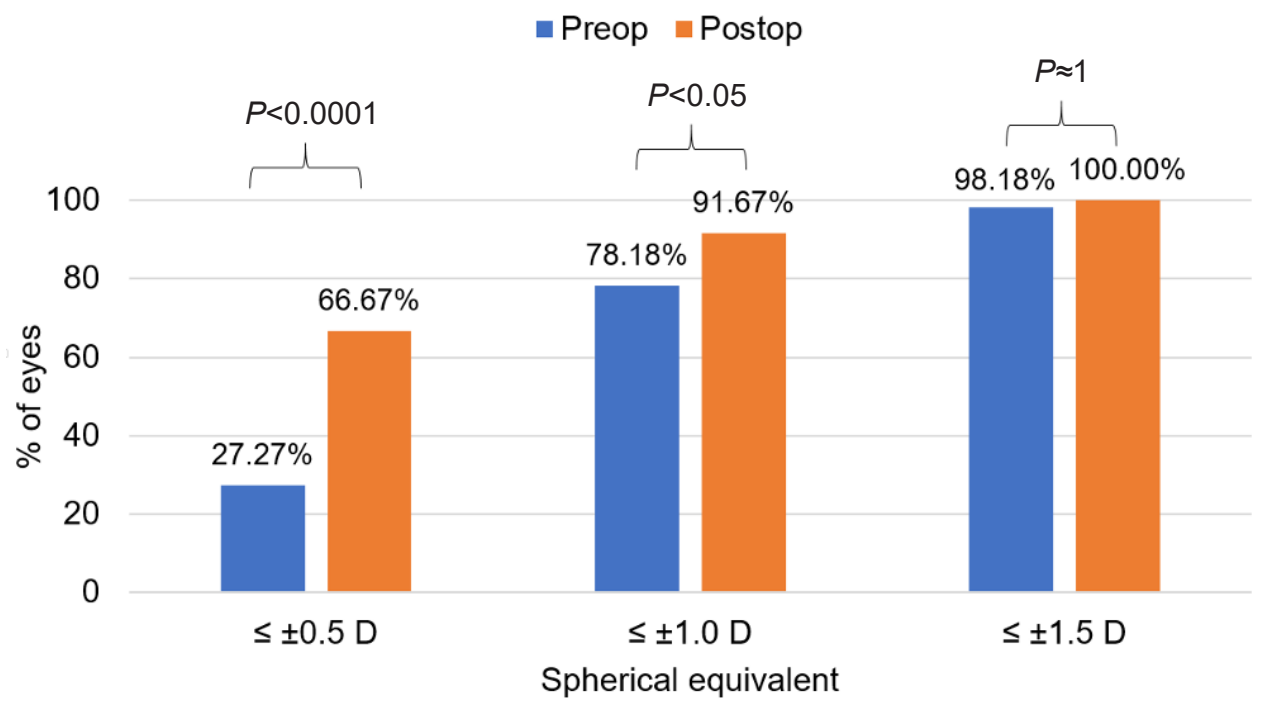

Fig. 1. Evaluation of spherical equivalent before and after laser enhancement. 


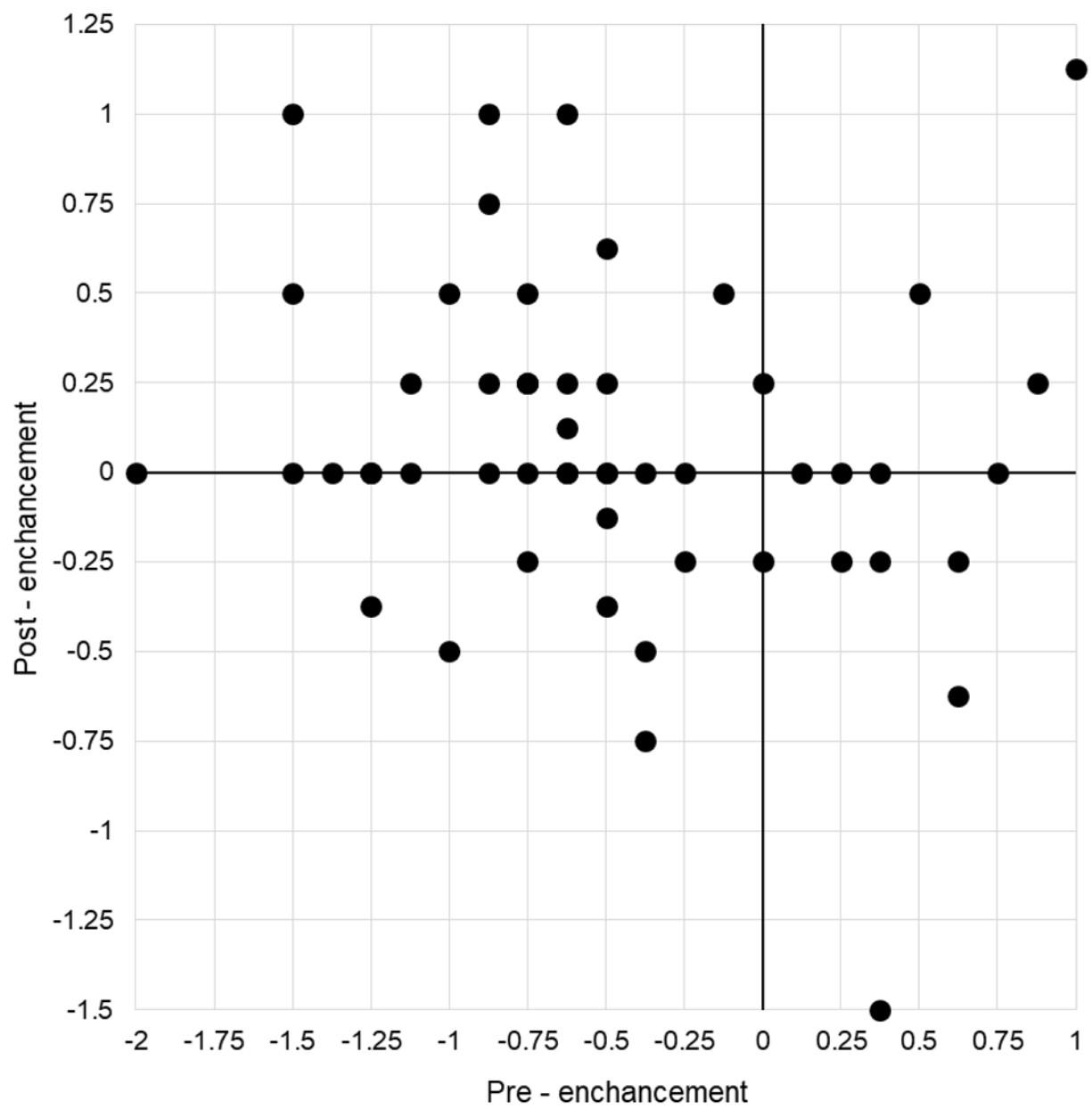

Fig. 2. Interdependence of pre-enhancement and post-enhancement spherical equivalent in diopters.

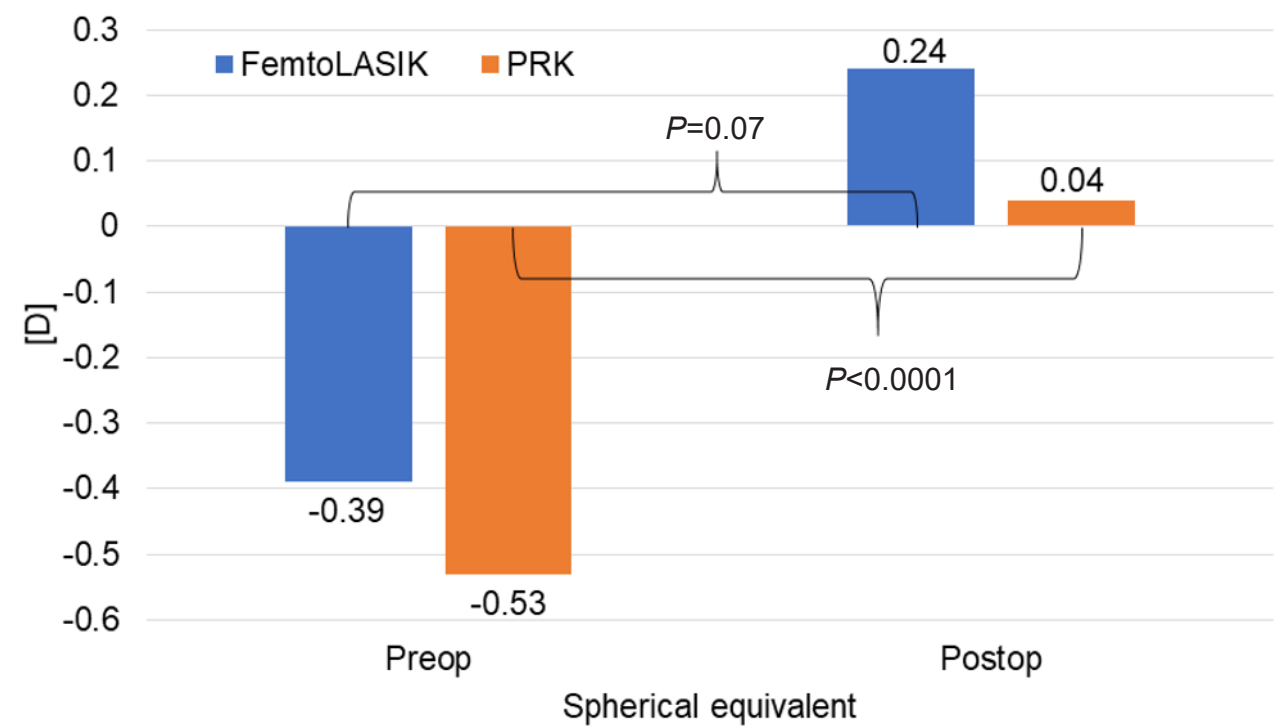

Fig. 3. Evaluation of spherical equivalent in diopters (D) in FemtoLASIK and PRK group.

Most optical biometry measurements are made using instruments that use highly accurate partial coherence interferometry but are some cases (like highly opaque crystalline lenses) where less accurate ultrasound biometry needs to be performed. In our study, two axial length measurements were performed because of high lens opacifica- tion. As we know, almost half of the errors in IOL power calculation using ultrasound biometry can be attributed to axial length measurement errors ${ }^{3}$. In both cases from our study, we performed laser retreatment.

Despite the effort to achieve the best results, undesired postoperative refractive error may occur. In general, 
postoperatively, patients with multifocal IOLs can be intolerant to even small degrees of ametropia, especially astigmatism $^{8}$.

Reported rates of enhancement after multifocal lens implantation in literature are significantly different. The multifocal retreatment rate in the retrospective study from Gundersen et al. ${ }^{9}$ was $10.8 \%$ (45 from 416 eyes), Mutfuoglu et al. ${ }^{10}$ report that 85 eyes from 246 eyes had LASIK to correct a residual refractive error (34.5\%) after multifocal IOL (AcrySof ReSTOR, Alcon) implantation. $99 \%$ of eyes were within $\pm 1.0 \mathrm{D}$ of emmetropia and $98 \%$ of eyes were within \pm 1 cylinder. These results are comparable to our outcomes $(5.4 \%)$, but the percentage of eyes treated after the primary procedure-cataract surgery or refractive lens exchange-in their study was much higher (34.5\%).

Schallhorn et al. ${ }^{1}$ presented outcomes of retrospective study of 782 eyes that underwent laser vision correction to correct unintended ametropia after implantation of several types of multifocal IOL. LASIK was performed on $91.2 \%$ of eyes, PRK in $8.8 \%$ of eyes. Postenhancement, $90.4 \%$ of eyes were within $0.5 \mathrm{D}$ and $99.5 \%$ were within 1.0 D of SE. This study presented a greater number of patients with a higher percentage of LASIK enhancements and slightly better refractive outcomes. The need for additional retreatment was similar in our studies, $5.9 \%$, and $4.92 \%$, respectively.

Artola et al. ${ }^{11}$ evaluated 30 eyes of 30 patients that had PRK to correct symptomatic residual myopia or myopic astigmatism after cataract surgery. Twelve months after the laser ablation, 22 eyes $(73 \%)$ were within $\pm 0.50 \mathrm{D}$ of emmetropia, 28 (93\%) within $\pm 1.00 \mathrm{D}$ and 29 (97\%) within $\pm 2.00 \mathrm{D}$. Two eyes $(6.66 \%)$ were re-treated for under-correction one year after PRK.

Fan et al. ${ }^{12}$ performed PRK in 18 consecutive eyes of 17 patients for correcting residual refractive error following cataract surgery with premium intraocular lens implantation. Six months after PRK, 14 eyes $(77.8 \%)$ showed a final SE within $\pm 0.50 \mathrm{D}$, and 17 eyes (94.4\%) within $\pm 1.00 \mathrm{D}$.

It has been observed that LASIK and PRK have similar efficacy and reliability for the correction of residual refractive error ${ }^{13}$. LASIK is a popular choice due to its fast and relatively pain-free recovery, but there could be an increased risk of dry eye symptoms with the creation of the corneal flap ${ }^{1}$. However, PRK is more suitable for patients with preexisting dry eye syndrome, decreased corneal thickness and irregular astigmatism ${ }^{13}$ with satisfactory and stable visual outcome ${ }^{14}$.

Gibbons et al. ${ }^{15}$ analyzed data from 74 eyes of 49 patients dissatisfied after presbyopia-correcting IOLs implantation. In $57 \%$, complaints were attributed to residual refractive error followed by dry eye in $35 \%$, causing blurry and foggy vision.

In general, it is essential to consider which method is optimal for the specific patient, the character of residual error, preferences and the experience of refractive surgeon. In our study, PRK was the preferred method for enhancement. We performed PRK in 50 patients $(82 \%)$ to solve postoperative myopia and mixed astigmatism. FemtoLASIK was used in specific cases to treat post- operative hyperopia and in younger patients with higher demands for faster healing time.

El-Agha et al. ${ }^{16}$ compared the efficacy and safety of PRK and laser in situ keratomileusis (LASIK) in the treatment of spherical hyperopia. The mean age for patients receiving PRK was $52 \pm 7$ years, in LASIK group $55 \pm 9$ years, which is similar to our study group. A review of 22 eyes receiving PRK and 26 eyes receiving LASIK showed, that these two methods are comparable in terms of efficacy and safety for low to moderate hyperopia. PRK was associated with significant postoperative pain, an initial and temporary myopic overshoot at one month and stability not occurring before 6 months after surgery. LASIK was less painful and was associated with more rapid stability at one month and better UCVA.

\section{CONCLUSION}

Our study showed that the implantation of FineVision Micro F trifocal lens is an effective solution for gaining independence from spectacle correction. With a properly guided and adequate preoperative strategy in the indication and selection of the dioptric power of the lens, the incidence of residual refractive error requiring laser enhancement is low.

There was no significant difference in visual outcomes between the FemtoLASIK and PRK groups, thus, any laser correction of residual refractive error is, according to our experience, a safe and effective solution for improving the quality of vision and patient satisfaction.

Presented at the 37th Annual Congress of the ESCRS, Paris, France, September 2019

Author contributions: DB: concept and design, supervision; DB, DJ, AK, MSA: data acquisition; DB, DJ: literature search; DJ: drafting manuscript; DB, MS, AK, MSA: critical revision of manuscript; MS: statistical analysis; DB, SJ, MS, AK, MSA: final approval.

Conflict of interest statement: None declared

\section{REFERENCES}

1. Schallhorn SC, Venter JA, Teenan D, Schallhorn JM, Hettinger KA, Hannan SJ, Pelouskova M. Outcomes of excimer laser enhancements in pseudophakic patients with multifocal intraocular lens. Clin Ophthalmol 2016;10:765-76.

2. Narvaez J, Zimmerman G, Stulting RD, Chang DH. Accuracy of intraocular lens power prediction using the Hoffer Q, Holladay 1, Holladay 2, and SRK/T formulas. J Cataract Refract Surg 2006;32:2050-53.

3. Olsen T. Sources of error in intraocular lens power calculation. J Cataract Refract Surg 1992;18:125-29.

4. Norrby S. Sources of error in intraocular lens power calculation. J Cataract Refract Surg 2008; 34:368-76.

5. Fernandez-Buenaga R, Alio JL, Perez Ardoy AL, Quesada AL, PinillaCortes $\mathrm{L}$, Barraquer RI. Resolving refractive error after cataract surgery: IOL exchange, piggyback lens, or LASIK. J Refract Surg 2013;29:676-83.

6. Kuo IC, O'Brien TP, Broman AT, Ghajarnia M, Jabbur NS. Excimer laser surgery for correction of ametropia after cataract surgery. J Cataract Refract Surg 2005;31:21042110. 
7. Kohnen T. Multifocal IOL technology: a successful step on the journey toward presbyopia treatment. J Cataract Refract Surg 2008;34:2005.

8. Woodward MA, Randleman JB, Stulting RD. Dissatisfaction after multifocal intraocular lens implantation. J Cataract Refract Surg 2009;35:992-97.

9. Gundersen KG, Makari S, Ostenstad S, Potvin R. Retreatments after multifocal intraocular lens implantation: an analysis. Clin Ophthalmol 2016;10:365-71.

10. Muftuoglu O, Prasher P, Chu C, Mootha VV, Verity SM, Cavanagh HD, Bowman RW, McCulley JP. Laser in situ keratomileusis for residual refractive errors after apodized diffractive multifocal intraocular lens implantation. J Cataract Refract Surg 2009;35:1063-71.

11. Artola A, Ayala MJ, Claramonte P, Perez-Santonja JJ, Alio JL. Photorefractive keratectomy for residual myopia after cataract surgery. J Cataract Refract Surg 1999;25:1456-60.
12. Fan YY, Sun CC, Chen HC, Ma DH. Photorefractive keratectomy for correcting residual refractive error following cataract surgery with premium intraocular lens implantation. Taiwan J Ophthalmol 2018:8:149-58.

13. Moshirfar M, McCaughey MV, Santiago-Caban L. Corrective Techniques and Future Directions for Treatment of Residual Refractive Error Following Cataract Surgery. Expert Rev Ophthalmol 2014;9:529-37.

14. Roszkowska AM, Urso M, Signorino GA, Spadea L, Aragona P. Photorefractive keratectomy after cataract surgery in uncommon cases: long-term results. Int J Ophthalmol 2018;11:612-15.

15. Gibbons A, Ali TK, Waren DP, Donaldson KE. Causes and correction of dissatisfaction after implantation of presbyopia-correcting intraocular lenses. Clin Ophthalmol 2016;10:1965-70.

16. el-Agha MS, Johnston EW, Bowman RW, Cavanagh HD, McCulley JP. Excimer laser treatment of spherical hyperopia: PRK or LASIK? Trans Am Ophthalmol Soc 2000;98:59-66; discussion 66-59. 